\title{
SpS1-Infrared and submillimetre-wave spectroscopy as probes of stellar evolution
}

\author{
Sun Kwok ${ }^{1}$ \\ ${ }^{1}$ Department of Physics, The University of Hong Kong, Hong Kong, China \\ email: sunkwok@hku.hk
}

\section{Introduction}

For over a hundred years, optical spectroscopy has been the main tool to study stellar structure and evolution. Photospheric spectra of the electronic transitions of atoms and ions are used to determine the temperature and elemental abundance. Beyond atomic and ionic lines, only the electronic transitions of a few simple molecules $\left(\mathrm{C}_{2}, \mathrm{CN}, \mathrm{H}_{2} \mathrm{O}, \mathrm{TiO}, \mathrm{CH}\right.$, etc. $)$ appear in the optical photospheric spectra. With the recent development of infrared and submm spectroscopy, a wide range of molecules have been observed, specially in cool atmospheres of red giants and brown dwarfs. We also realize that beyond the photosphere, a stellar system consists of chromosphere, corona, and stellar wind. Both young and evolved stars possess extensive circumstellar regions and the atoms, molecules, and solid particles in this environment radiate a wide range of lines and bands observable at infrared and submm wavelengths.

\section{Atoms and ions}

Many of the fine structure lines of common neutral atoms and ions lie in the infrared part of the spectrum. The ground state of neutral carbon (C I) has two fine-structure lines ${ }^{3} P_{2}-{ }^{3} P_{1}$ $(370 \mu \mathrm{m})$ and ${ }^{3} P_{1}-{ }^{3} P_{0}(609 \mu \mathrm{m})$. Neutral oxygen $(\mathrm{O}$ I $)$ has fine-structure lines ${ }^{3} P_{1}-{ }^{3} P_{2}(63$ $\mu \mathrm{m})$ and ${ }^{3} P_{0}-{ }^{3} P_{1}(145 \mu \mathrm{m})$. Other common fine-structure lines of ions include the ${ }^{2} P_{3 / 2}-{ }^{2} P_{1 / 2}$ line of $\mathrm{C}$ II at $158 \mu \mathrm{m}$, the ${ }^{3} P_{2}-{ }^{3} P_{1}(122 \mu \mathrm{m})$ and ${ }^{3} P_{1}-{ }^{3} P_{0}(205 \mu \mathrm{m})$ lines of $\mathrm{N}$ II, and the ${ }^{3} P_{2}-{ }^{3} P_{1}(52 \mu \mathrm{m})$ and ${ }^{3} P_{1}-{ }^{3} P_{0}(88 \mu \mathrm{m})$ of $[\mathrm{O} \mathrm{III}]$. Atoms with an odd number of nucleons can have hyperfine lines observable in the infrared. Examples are the $158 \mu \mathrm{m}{ }^{2} P_{3 / 2}-{ }^{2} P_{1 / 2} F=2-1$ line of $\left[{ }^{13} \mathrm{C} \mathrm{II}\right]$, the $9.0 \mu \mathrm{m}{ }^{3} P_{1}-{ }^{3} P_{2}$ line of [Na IV] and the $3.66 \mu \mathrm{m}$ line of $[\mathrm{Al} \mathrm{VI}]$.

\section{Molecules}

Since the development of $\mathrm{mm} / \mathrm{submm}$ receivers, the rotational transitions of over 60 molecules have been discovered in the circumstellar envelopes of evolved stars. These include inorganics (e.g., $\mathrm{CO}, \mathrm{SiO}, \mathrm{SiS}, \mathrm{NH}_{3}, \mathrm{AlCl}$, etc.), organics $\left(\mathrm{CH}_{4}, \mathrm{H}_{2} \mathrm{CO}, \mathrm{CH}_{3} \mathrm{CN}\right.$, etc), radicals $\left(\mathrm{CN}, \mathrm{C}_{2} \mathrm{H}\right.$, $\mathrm{C}_{3}, \mathrm{HCO}^{+}$, etc.), chains (e.g., $\mathrm{HCN}, \mathrm{HC}_{3} \mathrm{~N}, \mathrm{HC}_{5} \mathrm{~N}$, etc.), and rings $\left(\mathrm{C}_{3} \mathrm{H}_{2}\right)$. The rotational transitions of many of the lighter molecules lie in the submillimeter range. Some examples are the $N=2 \rightarrow 1^{2} \Pi_{3 / 2} \rightarrow^{2} \Pi_{1 / 2}$ at $149.390 \mu \mathrm{m}(-\rightarrow+)$ and $149.091 \mu \mathrm{m}(+\rightarrow-)$ of $\mathrm{CH}$, the ground-state ortho rotational transition $1_{10}-1_{01}(537.7 \mu \mathrm{m})$ of $\mathrm{H}_{2} \mathrm{O}$, the $(N, J)=3,3 \rightarrow 1,2$ $(615.7 \mu \mathrm{m})$ of $\mathrm{O}_{2}$, the $1_{11}-0_{00}(127.65 \mu \mathrm{m})$ line of methylene $\left(\mathrm{CH}_{2}\right)$.

Infrared spectroscopy from space has opened up the possibility of detecting the stretching and bending modes of molecules. For example, the fundamental vibrational mode of HCN consists of the stretching modes $\nu_{1}(100 \leftrightarrow 000)$ at $4.8 \mu \mathrm{m}$ and $\nu_{3}(001 \leftrightarrow 000)$ at $\left.3 \mu \mathrm{m}\right)$ and bending mode $\nu_{2}(010 \leftrightarrow 000)$ at $14 \mu \mathrm{m}$. The $13.7 \mu \mathrm{m} \nu_{5}$ cis-bend of acetylene $\left(\mathrm{C}_{2} \mathrm{H}_{2}\right)$ is commonly observed in absorption in extreme carbon stars. For $\mathrm{CO}_{2}$, in addition to the $01^{1} 0 \leftrightarrow 00^{0} 0$ fundamental $\nu_{2}$ bending mode at $14.97 \mu \mathrm{m}$, hot bends $(030 \leftrightarrow 020$ at $15.40 \mu \mathrm{m}, 020 \leftrightarrow 010$ at 16.18 $\mu \mathrm{m})$ and combination bands $(100 \leftrightarrow 010$ at $13.87 \mu \mathrm{m}$ and $110 \leftrightarrow 020$ at $13.48 \mu \mathrm{m})$ have been observed. $\mathrm{C}_{4} \mathrm{H}_{2}$ at $15.9 \mu \mathrm{m}, \nu_{11}$ of $\mathrm{C}_{6} \mathrm{H}_{2}$ at $\left.16.1 \mu \mathrm{m}\right), \nu_{4}$ of benzene $\left(\mathrm{C}_{6} \mathrm{H}_{6}\right)$ at $14.84 \mu \mathrm{m}$, and cyanopolyynes ( $\nu_{5}$ of $\mathrm{HC}_{3} \mathrm{~N}$ at $15.1 \mu \mathrm{m}, \nu_{7}$ of $\mathrm{HC}_{5} \mathrm{~N}$ at $15.6 \mu \mathrm{m}$ ) have been detected in absorption in the proto-planetary nebula AFGL 618. Pure carbon chains $\left(\mathrm{C}_{n}\right)$ have no permanent electricdipole moment and therefore have no permitted rotational transitions, but their stretching and bending modes can be observed in the infrared. For example, the stretching modes of $\mathrm{C}_{3}\left(\nu_{3}\right.$ at 
$4.90 \mu \mathrm{m})$ and $\mathrm{C}_{5}\left(\nu_{5}\right.$ at $\left.4.62 \mu \mathrm{m}\right)$ have been detected in absorption in carbon star spectra. The $\nu_{2}\left(0,1^{1}, 0\right) \leftrightarrow\left(0,0^{0}, 0\right)$ bending mode of $\mathrm{C}_{3}$ near $158 \mu \mathrm{m}$ can be observed with the Herschel Space Observatory. $J=2-0$ at $28.2188 \mu \mathrm{m})$ and ortho $\mathrm{H}_{2}(S(1), J=3-1$ at $17.0348 \mu \mathrm{m})$ have been observed by the Spitzer Space Telescope. Since HD is no longer homonuclear and has a dipole moment, the $v=0, J=1 \rightarrow 0, J=2 \rightarrow 1$, and $J=3 \rightarrow 2$ rotational transitions at 112.072, 56.2, and $37.7 \mu \mathrm{m}$ can be used to provide accurate determination of HD and to infer the deuterium abundance.

\section{Solids}

The $9.7 \mu \mathrm{m} \mathrm{Si}-\mathrm{O}$ stretching and the $18 \mu \mathrm{m} \mathrm{O}-\mathrm{Si}-\mathrm{O}$ bending modes of amorphous silicates are the most commonly observed solid-state features in stars, with over 4000 such spectra recorded by the IRAS Low Resolution Spectrometer. The O-H stretching mode $(3 \mu \mathrm{m})$ and bending mode $(6 \mu \mathrm{m})$ of amorphous water ice is also widely seen. Other common forms of ice features include the $4.67 \mu \mathrm{m} \mathrm{C}-\mathrm{O}$ stretch of $\mathrm{CO}$, the $15.3 \mu \mathrm{m} \mathrm{O}-\mathrm{C}-\mathrm{O}$ bend of $\mathrm{CO}_{2}$, the $7.7 \mu \mathrm{m}$ $\mathrm{C}-\mathrm{H}$ deformation mode of $\mathrm{CH}_{4}$. A family of infrared emission features at $3.3,6.2,7.7,8.6$, and $11.3 \mu \mathrm{m}$ have been identified as due to stretching and bending modes of aromatic compounds. Features at 3.4 and $6.9 \mu \mathrm{m}$ can be attributed to the $\mathrm{C}-\mathrm{H}$ stretching and bending modes of aliphatic units. Broad emission plateaus at 8 and $12 \mu \mathrm{m}$ are likely to be due to the in-plane and out-of-plane bending modes of a mixture of aliphatic groups attached to aromatic rings. Amorphous carbonaceous solids with mixed $s p^{2} / s p^{3}$ structures are now known to be rapidly synthesized in the circumstellar environment of evolved stars.

\section{Conclusions}

With new observing facilities such as Herschel, SOFIA, and $A L M A$, we will have powerful tools in the infrared and submm wavelengths to probe the physical and chemical structures of stellar environments.

\section{Acknowledgements}

This work is supported by a grant from the Research Grants Council of the Hong Kong Special Administrative Region, China (Project No. HKU 7020/08P).

\section{References}

Kwok, S. 2007, Physics and Chemistry of the Interstellar Medium, University Science Books 